\title{
Slab analysis of asymmetrical sheet rolling
}

\author{
Shunhu Zhang ${ }^{1, a}$, Meng. Wei ${ }^{2, b}$, Xinyi Wang ${ }^{1, a}$ and Min Liu ${ }^{1, a}$ \\ ${ }^{1}$ Shagang School of Iron and Steel, Soochow University, Suzhou 215021, China \\ ${ }^{2}$ Law school, Jiangnan University, Wuxi 214122, China \\ ashzhang@suda.edu.cn, ${ }^{\mathrm{b}} 563654070 @ q q . c o m$
}

Keywords: asymmetrical sheet rolling; slab method; analytical solution; cross shear zone

\begin{abstract}
An analytical solution based on slab method is first proposed to calculate the rolling force and rolling torque in asymmetrical sheet rolling where the work roll radii, their speeds, and the interfacial frictions may be different. In the solution, the shear stress acting along the vertical sides of each slab is taken into account. To verify the validity of the proposed analytical solution, the analytical rolling force and torque were compared with experimental and analytical results of other investigators. Very good agreements are found. By this proposed analytical solution, it is noted that the calculation time and computer expense are saved, and the characteristics of asymmetrical cold and hot sheet rolling are obtained easily and rapidly.
\end{abstract}

\section{Introduction}

Asymmetrical sheet rolling processes, for which the peripheral velocity or radius of the upper roll may be different from those of the lower roll, have become more and more important, in light of the fact that it can gain such advantages as lower rolling pressure distribution, resulting in less rolling force. Sachs and Klinger (1947) seem to be the early investigators in this area who concentrated on the mechanics of single roll driven mills [1]. They realized that there exists a region identified as the region of cross shear where the frictional forces on the driven and undriven rolls act on the strip in opposite directions and the developed curvature at exit is due to this region. Pan and Sansome (1982) carried out some experiments on asymmetric rolling, in which asymmetry was created due to speed mismatch. In progressive analytical studies, Hwang and Tzou (1997) attempted to analyze the asymmetrical rolling process [3]. In their models, only uniform normal stress in the absence of any shear stress was assumed to act on the vertical sides of each slab, and the effect of frictional shear stresses in deriving the yield criterion was neglected. Salimi and Kadkhodaei (2004) used a new stress field in which non-uniform normal and shear stresses on the vertical sides of each slab were considered, and frictional shear stresses in evaluating the yield criteria were taken into account [4]. In their investigation, they stumbled on that the sheet may tilt at entry if it is fed freely. Consequently to have horizontal entry of the sheet, it is needed to impose extra techniques that warranted horizontal entry of the sheet into the roll gap. Kadkhoadei et al. (2007) used a genetic algorithm to calculate deflection and the angle of rotation in the ingoing sheet [5]. These models were able to evaluate the rolling force and torque. Gudur et al. (2008) [6] improved Salimi and Kadkhodaei [7] approach by incorporating strain-hardening effects to estimate friction in asymmetrical sheet rolling with no force and moment at entry by calculating the strip curvature at exit. Qwamizade et al. (2011) evaluated the developed curvature during asymmetrical sheet rolling in which quadratic shear stress and linear normal stress distributions were assumed to act on the vertical sides of the slab [8].

Most of the above mentioned researches, except for the analytical solution obtained by Hwang [3] in which the shear stress on the vertical sides of each slab was not considered and the maximum error may reach up to $15 \%$ for the rolling pressure, on the rolling pressure distribution, rolling force, rolling torque, as well as the strip curvature at exit, were numerical. Using numerical method such as the Runge-Kutta method to solve the governing equations in the analysis of asymmetrical rolling is time-consuming and inconvenient. A large number of divided intervals are needed to get accurate solutions. Thus, obtaining an analytical solution which accounts for the shear stress on the vertical 
sides of each slab and can accurately predict the rolling force and torque is still necessary, and that is the main objective of this paper.

Up to now, recent researches either focus on the numerical solutions taking the shear stress on the vertical sides of each slab into account or stay in the analytical solutions neglecting the shear stress. In the present work, an analytical solution based on the slab method for asymmetrical cold rolling is first proposed, in which the shear stresses on the vertical sides of each slab have been incorporated in deriving the yield criterion. What's more, effects of the roll speed ratio, the friction factor, and the friction factor ratio, on the rolling pressure distribution, rolling force and rolling torque are discussed systematically.

\section{Mathematical Model}

In the development of the mathematic model to be presented, the following assumptions are made:

(1) The work rolls are assumed to be rigid and the sheet to be rigid perfectly plastic with no strain-hardening effect. (2) The plastic deformation is plane strain and no material spread in transverse direction is taken into account. (3) The normal stress on the vertical sides of each slab is uniform and the interfacial shear stresses $\tau_{u}$ and $\tau_{l}$ at the upper and the lower interfaces are constant and follow Tresca's law, i.e. $\tau_{u}=m_{u} k, \tau_{l}=m_{l} k$. (4) All the shear stresses on the vertical side of the element in the three distinct zones will reach their maximum values $\tau=m k$ at the upper and lower surfaces. However, their distributions are different for the three distinct zones. In zones I (backward slip zone) and III (forward slip zone) the shear stresses will obtain its minimum values of zero at the center, and the shear stresses from the upper and lower surfaces to their center are linearly distributed. But, in zone II (cross shear zone) the shear stresses from the upper surface to the lower surface are linearly distributed and the shear stress at the center is equal to that of the mean value of its surfaces. (5) The flow directions of the sheet at the entrance and exit of the roll gap are both horizontal. So, no curvature calculation is possible. (6) The total roll contact arc is comparatively smaller than the circumference of the rolls.

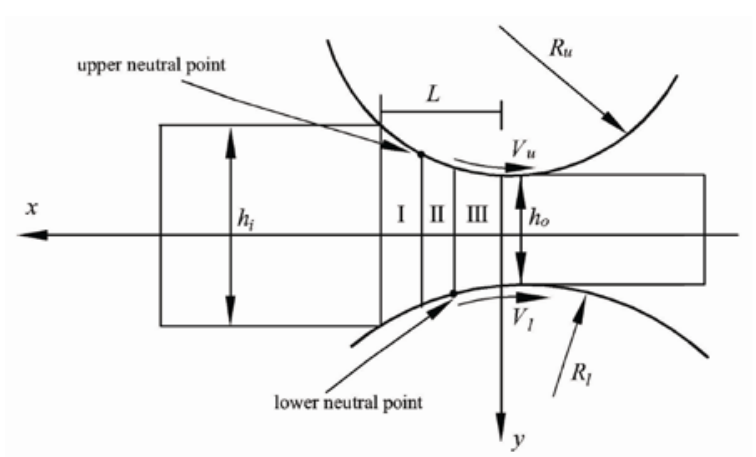

Fig. 1 Schematic illustration of the mathematical model

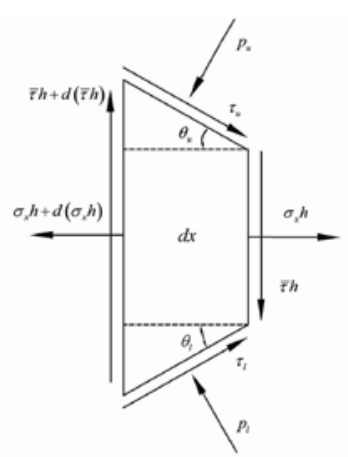

Fig. 2 Material element in region I

Fig. 1 shows the schematic diagram of the asymmetric rolling process. The inlet and outlet thickness of the strip are denoted by $h_{i}$ and $h_{o}$, respectively. The subscripts $u$ and $l$ represent upper and lower interfaces, respectively. Thus, $R_{u}$ and $R_{l}$ denote the radii of the upper and lower rolls whereas $V_{u}$ and $V_{l}$ represent the speeds of the upper and the lower rolls. Without any loss of generality, assume that the speed of the lower roll is higher than that of the upper roll. In Fig. 1, the roll gap is divided into three zones. In zone I, the strip velocity is lower than that of both the rolls and the frictional stresses on the upper and the lower surfaces are in the forward direction. In zone II, the strip velocity is more than the speed of the upper roll and less than the speed of the lower roll. Hence, the frictional stresses on the upper surface act in the backward direction and that on the lower surface act in the forward direction. In zone III, as the speeds of the two work rolls are lower than the strip velocity, the frictional stresses at both of the surfaces are in the backward direction. 
The stress state of a slab in zone I is illustrated in Fig. 2, in which the directions of the upper and lower friction forces are both forward, i.e., the velocities of the upper and lower rolls are both higher than that of the sheet. The horizontal position of the neutral point on the upper roll is not necessarily equal to that on the lower roll. Therefore, the direction of friction force from the upper roll exerted on the sheet is not necessarily the same as that from the lower roll. It is noted that in this present stress field in Fig. 2 the shear stress acted on the vertical side has been taken into account.

If we consider the equilibriums of a small element with sides parallel to $x$ and $y$ axes at the upper left limit and lower left limit of the material elements in the three distinct zones, then the assumed shear stress distribution (including the values and signs of the shear stresses) on the vertical side can be displayed in Fig. 3. Accordingly, the average shear stresses in zones I, II, and III can be calculated as $\bar{\tau}=\frac{1}{4}\left(m_{u}-m_{l}\right) k, \bar{\tau}=\frac{1}{2}\left(m_{u}+m_{l}\right) k$ and $\bar{\tau}=\frac{1}{4}\left(m_{l}-m_{u}\right) k$.

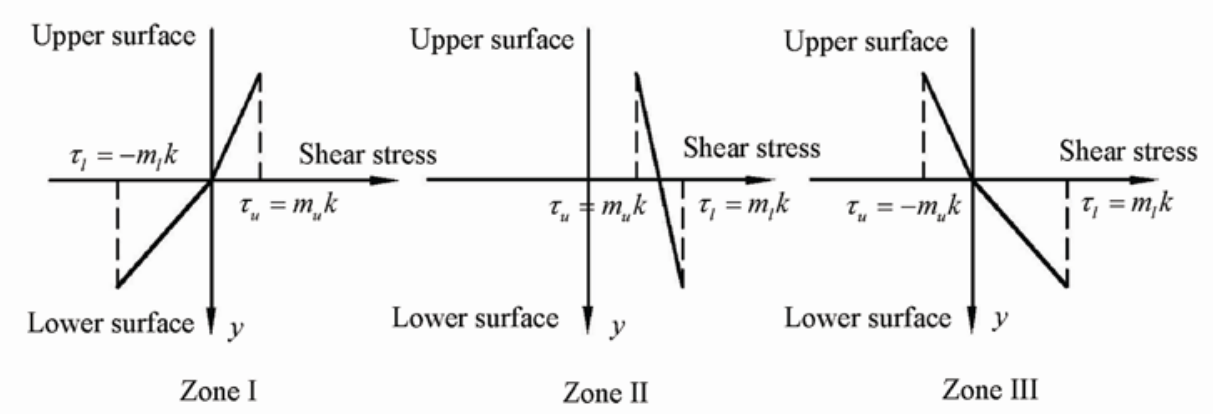

Fig. 3 Assumed shear stress distribution in zone I, II and III

Equilibrium Differential Equation. In Fig. 2, the normal stress is assumed to be distributed uniformly, i.e. $\sigma_{x}=\sigma_{u}=\sigma_{l}$, and the average shear stress $(\bar{\tau})$ is constant. As the contact length in comparison to the rolls radii is small we have:

$$
h=h_{o}+\frac{x^{2}}{R_{e q}}, R_{e q}=\frac{2 R_{u} R_{l}}{R_{u}+R_{l}}, \frac{d h}{d x}=\frac{2 x}{R_{e q}}
$$

where $R_{e q}$ is the equivalent work roll radius.

Without any loss of generality, we take the zone I as the analysis object firstly. For small reduction per pass $\tan \theta_{u}=x / R_{u}$ and $\tan \theta_{l}=x / R_{l}$, then the horizontal force equilibrium takes the form

$$
\sum F_{x}=0 \Rightarrow \frac{2 \sigma_{x} x}{R_{e q}}+h d \sigma_{x}+\frac{p_{u} x}{R_{u}}+\frac{p_{l} x}{R_{l}}-\tau_{e}=0 ; \tau_{e}=\tau_{u}+\tau_{l}
$$

where $p_{u}$ and $p_{l}$ are the rolling pressures of the upper and lower rolls, respectively.

Referring to Fig. 2, the mathematical expressions for vertical force equilibrium for zones I-III, for small bite angle is

$$
p=-\sigma_{y u}=-\sigma_{y l}=p_{u}+\tau_{u} \frac{x}{R_{u}}=p_{l}+\tau_{l} \frac{x}{R_{l}}
$$

where $p$ is the vertical stress at the roll gap.

The flow rule and the plane strain condition at any point in the plastic region gives

$$
\tau_{x z}=\tau_{y z}=0, \sigma_{z}=\frac{1}{2}\left(\sigma_{x}+\sigma_{y}\right)
$$

Substituting these relations into the Von-Mises yield criterion gives 


$$
\left|\frac{\sigma_{x}-\sigma_{y}}{2}\right|=\sqrt{k^{2}-\tau_{x y}^{2}}
$$

Considering $\sigma_{y}=\left(\sigma_{y u}+\sigma_{y l}\right) / 2=-p$ as the average vertical stress in $y$ direction and using the average shear stress $\bar{\tau}$ instead of $\tau_{x y}$ yields

$$
\sigma_{x}+p=2 \sqrt{k^{2}-\bar{\tau}^{2}}=2 k \sqrt{1-(\bar{\tau} / k)^{2}}=2 n_{\tau} k ; n_{\tau}=\sqrt{1-(\bar{\tau} / k)^{2}}
$$

where $n_{\tau}$ is defined as the shear stress factor which reflects the effect of average vertical shear stress on the mean yield shear stress.

Taking notice of $d \sigma_{x}=-d p$ and substituting Eqs. (3) and (6) into Eq. (2) and rearranging we obtain

$$
h \frac{d p}{d x}=-\left(\frac{\tau_{u}}{R_{u}^{2}}+\frac{\tau_{u}}{R_{l}^{2}}\right) x^{2}+\frac{4 n_{\tau} k}{R_{e q}} x-\tau_{e}
$$

Integrating Eq. (7) with respect to $x$, the solution of the differential equation is obtained as

$$
p=-A x+2 n_{\tau} k \ln \left(x^{2}+R_{e q} h_{o}\right)+\frac{B}{\sqrt{R_{e q} h_{o}}} \eta(x)+c^{*}
$$

where $A=R_{e q}\left(\frac{\tau_{u}}{R_{u}^{2}}+\frac{\tau_{l}}{R_{l}^{2}}\right), B=R_{e q} h_{o} A-R_{e q} \tau_{e}, \eta(x)=\arctan \frac{x}{\sqrt{R_{e q} h_{o}}}$, and $c^{*}$ is a constant of integration.

It should be noted that since the directions of friction stresses ( $\tau_{u}$ and $\tau_{l}$ in $\tau_{e}$ ) have already been considered in Eq. (1), and taking notice of the definition of $\tau_{e}=\tau_{u}+\tau_{l}$, then the friction stress toward forward (or rightwards) should be positive, and vice versa. In zone I, the directions of the friction forces are forward, i.e., the sheet velocity is slower than those of the upper and lower rolls, accordingly the equivalent friction stress $\tau_{e}$ is $m_{u} k+m_{l} k$. The form of the differential equation in III is the same as that in zone I, except that the effective friction stress $\tau_{e}$ is replaced by $\tau_{e}=-m_{u} k-m_{l} k=-\left(m_{u}+m_{l}\right) k$, because the directions of the friction forces are backward (or leftwards). In zone II, the friction forces are in reverse direction, thus $\tau_{e}=-m_{u} k+m_{l} k=\left(m_{l}-m_{u}\right) k$ for the case of $V_{l}>V_{u}$.

Boundary Conditions. The velocity of the lower roll is assumed to be quicker than that of the upper roll. The neutral point of the upper roll is denoted by $x_{n u}$, and that the lower of roll by $x_{n l}$. The boundary conditions for those three distinct zones can be expressed as follows:

(i) Zone III $\left(0 \leq x \leq x_{n l}\right), \tau_{e 3}=-\left(m_{u}+m_{l}\right) k, \quad \bar{\tau}_{3}=\frac{1}{4}\left(m_{u}-m_{l}\right) k, n_{\tau_{3}}=\sqrt{\left[1-\left(m_{u}-m_{l}\right)^{2} / 16\right]}$

At $x=0[$ or $\eta(x)=0]$

$$
p_{0}=2 n_{\tau_{3}} k-\sigma_{x 0}
$$

where $p_{0}$ is the rolling pressure at the exit of the roll gap and $\sigma_{x 0}$ is the front tension at the exit. From this boundary condition, integral constant $c^{*}$ in Eq. (8) can be obtained as

$$
c_{3}^{*}=2 n_{\tau_{3}} k\left[1-\ln \left(R_{e q} h_{o}\right)\right]-\sigma_{x 0}
$$


where the subscript 3 in variables $\tau_{e 3}, \bar{\tau}_{3}, n_{\tau_{3}}$ and $c_{3}^{*}$ denote the $\tau_{e}, \bar{\tau}, n_{\tau}$ and integral constant $c^{*}$ in zone III. Hence, the rolling pressure $\left(p_{\text {III }}\right)$ in zone III can be expressed as

$$
p_{\text {III }}=-A_{3} x+2 n_{\tau_{3}} k \ln \left(x^{2}+R_{e q} h_{0}\right)+\frac{B_{3}}{\sqrt{R_{e q} h_{0}}} \eta(x)+c_{3}^{*}
$$

where $A_{3}=-R_{e q} k\left(\frac{m_{u}}{R_{u}^{2}}+\frac{m_{l}}{R_{l}^{2}}\right), B_{3}=R_{e q} h_{o} A_{3}-R_{e q} \tau_{e 3}$.

(ii) Zone I $\left(x_{n u} \leq x \leq L\right), \tau_{e 1}=\left(m_{1}+m_{2}\right) k, \bar{\tau}_{1}=\frac{1}{4}\left(m_{u}+m_{l}\right) k, n_{\tau_{1}}=\sqrt{\left[1-\left(m_{u}+m_{l}\right)^{2} / 16\right]}$

At $x=L\left[\right.$ or $\left.\eta(L)=\arctan \left(L / \sqrt{R_{e q} h_{o}}\right)\right]$

$$
p_{1}=2 n_{\tau_{1}} k-\sigma_{x 1}
$$

where $p_{1}$ is the rolling pressure at the entrance of the roll gap and $\sigma_{x 1}$ is the back tension at the entrance. From this boundary condition, $c_{1}^{*}$ can be expressed as

$$
c_{1}^{*}=2 n_{\tau_{1}} k-\sigma_{x 1}+A_{1} L-2 n_{\tau_{1}} k \ln \left(L^{2}+R_{e q} h_{o}\right)-\frac{B}{\sqrt{R_{e q} h_{0}}} \eta(L)
$$

where $A_{1}=R_{e q} k\left(\frac{m_{u}}{R_{u}^{2}}+\frac{m_{l}}{R_{l}^{2}}\right), B_{1}=R_{e q} h_{o} A_{1}-R_{e q} \tau_{e 1}, L=\sqrt{R_{e q} h_{i} r}$.

Therefore, the rolling pressure $\left(p_{\mathrm{I}}\right)$ is as follows

$$
p_{\mathrm{I}}=-A_{1} x+2 n_{\tau_{1}} k \ln \left(x^{2}+R_{e q} h_{o}\right)+\frac{B_{1}}{\sqrt{R_{e q} h_{o}}} \eta(x)+c_{1}^{*}
$$

When the peripheral velocity of the upper roll $\left(V_{u}\right)$ is smaller than that of the lower roll $\left(V_{l}\right)$, the margin of $x$ in zone II is $x_{n l} \leq x \leq x_{n u}$, and $\tau_{e 2}=-m_{u} k+m_{l} k=\left(m_{l}-m_{u}\right) k$.

(iii) Zone II $\left(x_{n l} \leq x \leq x_{n u}\right), \tau_{e 2}=\left(m_{l}-m_{u}\right) k, \quad \bar{\tau}_{2}=\frac{1}{2}\left(m_{u}+m_{l}\right) k, n_{\tau_{2}}=\sqrt{\left[1-\left(m_{u}+m_{l}\right)^{2} / 4\right]}$

Due to the continuity of boundary conditions at $x=x_{\text {nl }}$, the rolling pressure in zone III $\left(p_{\text {III }}\right)$ at $x=x_{n l}$ has to be equal to that in zone II $\left(p_{\text {II }}\right)$, i. e. $p_{\text {III }}=p_{\text {II }}$. Accordingly, $c_{3}^{*}$ and $c_{2}^{*}$ have the relationship as

$$
-A_{3} x_{n l}+2 n_{\tau_{3}} k \ln \left(x_{n l}^{2}+R_{e q} h_{o}\right)+\frac{B_{3}}{\sqrt{R_{e q} h_{o}}} \eta\left(x_{n l}\right)+c_{3}^{*}=-A_{2} x_{n l}+2 n_{\tau_{2}} k \ln \left(x_{n l}^{2}+R_{e q} h_{o}\right)+\frac{B_{2}}{\sqrt{R_{e q} h_{o}}} \eta\left(x_{n l}\right)+c_{2}^{*}
$$

Where $A_{2}=-R_{e q} k\left(\frac{m_{u}}{R_{u}^{2}}-\frac{m_{l}}{R_{l}^{2}}\right), B_{2}=R_{e q} h_{o} A_{2}-R_{e q} \tau_{e 2}$.

Meanwhile, due to the continuity of boundary conditions at $x=x_{n u}$, i.e. $p_{\mathrm{I}}=p_{\mathrm{II}}$, we can obtain

$$
-A_{1} x_{n u}+2 n_{\tau_{1}} k \ln \left(x_{n u}^{2}+R_{e q} h_{o}\right)+\frac{B_{1}}{\sqrt{R_{e q} h_{o}}} \eta\left(x_{n u}\right)+c_{1}^{*}=-A_{2} x_{n u}+2 n_{\tau 2} k \ln \left(x_{n u}^{2}+R_{e q} h_{o}\right)+\frac{B_{2}}{\sqrt{R_{e q} h_{o}}} \eta\left(x_{n u}\right)+c_{2}^{*}
$$


where $\eta\left(x_{n u}\right)=\arctan \frac{x_{n u}}{\sqrt{R_{e q} h_{o}}}, \eta\left(x_{n l}\right)=\arctan \frac{x_{n l}}{\sqrt{R_{e q} h_{o}}}$.

From Eq. (13), $c_{2}^{*}$ can be expressed as

$c_{2}^{*}=\left(A_{2}-A_{3}\right) x_{n l}+F \eta\left(x_{n l}\right)+2\left(n_{\tau_{3}}-n_{\tau_{2}}\right) k \ln \left(x_{n l}^{2}+R_{e q} h_{o}\right)+c_{3}^{*}$

where $F=\left(B_{3}-B_{2}\right) / \sqrt{R_{e q} h_{o}}$.

and from Eq. (14), $c_{2}^{*}$ can be determined as

$c_{2}^{*}=\left(A_{2}-A_{1}\right) x_{n u}+E^{*} \eta\left(x_{n u}\right)+2\left(n_{\tau_{1}}-n_{\tau_{2}}\right) k \ln \left(x_{n u}^{2}+R_{e q} h_{o}\right)+c_{1}^{*}$

where $E=\left(B_{1}-B_{2}\right) / \sqrt{R_{e q} h_{o}}$.

Substituting Eq. (16) into Eq. (15) then produces

$$
\begin{aligned}
& \left(A_{2}-A_{1}\right) x_{n u}+E^{*} \eta\left(x_{n u}\right)+2\left(n_{\tau_{1}}-n_{\tau_{2}}\right) k \ln \left(x_{n u}^{2}+R_{e q} h_{o}\right)+c_{1}^{*} \\
& -\left(A_{2}-A_{3}\right) x_{n l}-F \eta\left(x_{n l}\right)-2\left(n_{\tau_{3}}-n_{\tau_{2}}\right) k \ln \left(x_{n l}^{2}+R_{e q} h_{o}\right)-c_{3}^{*}=0
\end{aligned}
$$

From the volume constancy of the material, the positions of the upper and lower neutral points $x_{n u}$ and $x_{n l}$, have the following relationship as [3]

$$
x_{n u}=\sqrt{V_{A} x_{n l}^{2}+\left(V_{A}-1\right) \frac{h_{o}}{R_{A}}} ; V_{A}=\frac{V_{l}}{V_{u}}, R_{A}=\frac{1}{R_{e q}}-\frac{h_{o}}{2 R_{e q}^{2}} .
$$

Substituting Eq. (18) into Eq. (17), the solution of the neutral point $x_{n l}$ can be easily found by the bisection numerical method. Once $x_{n l}$ is known, $x_{n u}$ and $c_{2}^{*}$ can be steadily obtained by Eqs. (18) and (15), respectively. The rolling pressure $\left(p_{\text {II }}\right)$ in zone II can be determined as

$$
p_{\text {II }}=-A_{2} x+2 n_{\tau_{2}} k \ln \left(x^{2}+R_{e q} h_{o}\right)+\frac{B_{2}}{\sqrt{R_{e q} h_{o}}} \eta(x)+c_{2}^{*}
$$

The rolling pressure $p_{\mathrm{I}}, p_{\mathrm{II}}$, and $p_{\mathrm{III}}$ can be calculated from Eqs. (12), (19) and (10), respectively, when $c_{1}^{*}, c_{2}^{*}$ and $c_{3}^{*}$ are known from Eqs. (11), (15) and (9), respectively.

Rolling Force and Torque. Once the mean shear yield strength of the material and friction factor between the rolls and sheet are known, the rolling force can be found by integrating the normal rolling pressure over the arc length of contact. Thus the rolling force per unit width is given as

$$
P=P_{\mathrm{I}}+P_{\mathrm{II}}+P_{\mathrm{III}}
$$

where

$$
P_{\mathrm{III}}=\int_{0}^{x_{n l}} p_{\mathrm{III}} d x=\mathrm{III}_{1}^{*}+\mathrm{III}_{2}^{*}
$$




$$
\begin{aligned}
& \mathrm{III}_{1}^{*}=-\frac{A_{3}}{2} x_{n l}^{2}+2 n_{\tau_{3}} k x_{n l} \ln \left(x_{n l}^{2}+R_{e q} h_{o}\right)+\left(c_{3}^{*}-4 n_{\tau_{3}} k\right) x_{n l}+4 n_{\tau_{3}} k \sqrt{R_{e q} h_{o}} \eta\left(x_{n l}\right) \\
& +B_{3}\left[\frac{x_{n 2}}{\sqrt{R_{e q} h_{o}}} \eta\left(x_{n l}\right)-\frac{1}{2} \ln \left(R_{e q} h_{o}+x_{n l}^{2}\right)\right] \\
& \mathrm{III}_{2}^{*}=\frac{B_{3}}{2} \ln \left(R_{e q} h_{o}\right) \\
& P_{\mathrm{II}}=\int_{x_{n l}}^{x_{n u}} p_{\mathrm{II}} d x=\mathrm{II}_{1}^{*}+\mathrm{II}_{2}^{*} \\
& \mathrm{II}_{1}^{*}=-\frac{A_{2}}{2} x_{n u}^{2}+2 n_{\tau_{2}} k x_{n u} \ln \left(x_{n u}^{2}+R_{e q} h_{o}\right)+\left(c_{2}^{*}-4 n_{\tau_{2}} k\right) x_{n u}+4 n_{\tau_{2}} k \sqrt{R_{e q} h_{o}} \eta\left(x_{n u}\right) \\
& +B_{2}\left[\frac{x_{n u}}{\sqrt{R_{e q} h_{o}}} \eta\left(x_{n u}\right)-\frac{1}{2} \ln \left(R_{e q} h_{o}+x_{n u}^{2}\right)\right] \\
& \mathrm{II}_{2}^{*}=\frac{A_{2}}{2} x_{n l}^{2}-2 n_{\tau_{2}} k x_{n l} \ln \left(x_{n l}^{2}+R_{e q} h_{o}\right)-\left(c_{2}^{*}-4 n_{\tau_{2}} k\right) x_{n l}-4 n_{\tau_{2}} k \sqrt{R_{e q} h_{o}} \eta\left(x_{n l}\right) \\
& -B_{2}\left[\frac{x_{n l}}{\sqrt{R_{e q} h_{o}}} \eta\left(x_{n l}\right)-\frac{1}{2} \ln \left(R_{e q} h_{o}+x_{n l}^{2}\right)\right] \\
& P_{\mathrm{I}}=\int_{x_{n u}}^{L} p_{\mathrm{I}} d x=\mathrm{I}_{1}^{*}+\mathrm{I}_{2}^{*} \\
& \mathrm{I}_{1}^{*}=-\frac{A_{1}}{2} L^{2}+2 n_{\tau_{1}} k L \ln \left(L^{2}+R_{e q} h_{o}\right)+\left(c_{1}^{*}-4 n_{\tau_{1}} k\right) L+4 n_{\tau_{1}} k \sqrt{R_{e q} h_{o}} L+B_{1}\left[\frac{L}{\sqrt{R_{e q} h_{o}}} \eta(L)-\frac{1}{2} \ln \left(R_{e q} h_{o}+L^{2}\right)\right] \\
& I_{2}^{*}=\frac{A_{1}}{2} x_{n u}^{2}-2 n_{\tau_{1}} k x_{n u} \ln \left(x_{n u}^{2}+R_{e q} h_{o}\right)-\left(c_{1}^{*}-4 n_{\tau_{1}} k\right) x_{n u}-4 n_{\tau_{1}} k \sqrt{R_{e q} h_{o}} \eta\left(x_{n u}\right) \\
& -B_{1}\left[\frac{x_{n u}}{\sqrt{R_{e q} h_{o}}} \eta\left(x_{n u}\right)-\frac{1}{2} \ln \left(R_{e q} h_{o}+x_{n u}^{2}\right)\right]
\end{aligned}
$$

The rolling torques, $T_{u}$ and $T_{l}$, exerted by the sheet on the upper and lower rolls, respectively, can be calculated by integrating the moment of the shear friction force along the arc length of contact around the roll axis. Therefore

$$
\begin{aligned}
& T_{u}=R_{u}\left(-\int_{0}^{x_{n l}} m_{u} k d x-\int_{x_{n l}}^{x_{n u}} m_{u} k d x+\int_{x_{n u}}^{L} m_{u} k d x\right)=R_{u} m_{u} k\left(L-2 x_{n u}\right) \\
& T_{l}=R_{l}\left(-\int_{0}^{x_{n l}} m_{l} k d x+\int_{x_{n l}}^{x_{n u}} m_{l} k d x+\int_{x_{n u}}^{L} m_{l} k d x\right)=R_{l} m_{l} k\left(L-2 x_{n l}\right)
\end{aligned}
$$


and the total torque required is

$T=T_{u}+T_{l}$.

\section{Results and Discussion}

The present model is compared for the rolling force values with the analytical and experimental results of Hwang and Tzou [3] as well as numerical results obtained by Salimi et al. [8], shown in Fig. 4. It shows that the results of the present model are in a better agreement with the experimental ones than those of Hwang model. It should be noted that although the present model is less accurate than the numerical model by Salimi et al., the present model is an analytical solution which can easily and quickly predict the characteristics in asymmetrical rolling and can save computational cost. What's more, the rolling forces for the three models all increase as the percentage reductions increase.

(a)

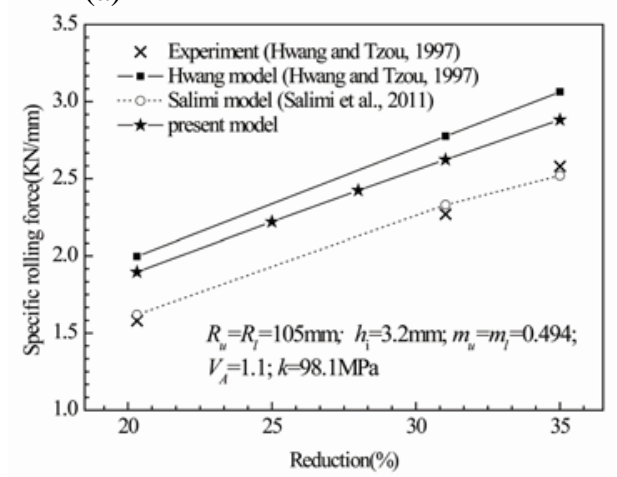

(b)

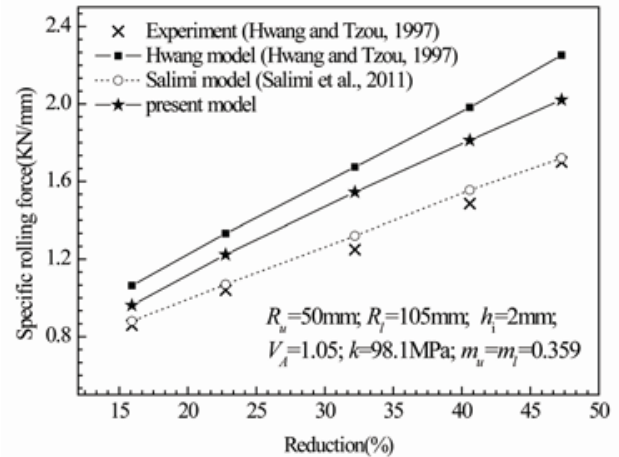

Fig. 4 Comparison of rolling force predicted by the present model with other researchers' results for (a) $\left(R_{u}=R_{l}=105 \mathrm{~mm} ; h_{i}=3.2 \mathrm{~mm} ; m_{u}=m_{l}=0.494 ; V_{A}=1.1 ; k=98.1 \mathrm{Ma}\right),(\mathbf{b})\left(R_{u}=50 \mathrm{~mm} ; R_{l}=105 \mathrm{~mm} ; h_{i}=2 \mathrm{~mm} ;\right.$ $\left.m_{u}=m_{l}=0.359 ; V_{\mathrm{A}}=1.05 ; k=98.1 \mathrm{Ma}\right)$

Fig. 5(a) shows the effect of roll speed ratio $V_{A}$ on the specific rolling force. The results of the rolling force models by Hwang and Tzou [3] and Salimi and Kadkhodaei [4] are also shown in this figure. It shows that all the rolling forces decrease as the roll speed ratio increases. Both the relationship between the rolling force and speed mismatch is quadratic curve for the present model and Hwang's model. Besides, the present result is much closer to that of Salimi model than by Hwang model.

(a)

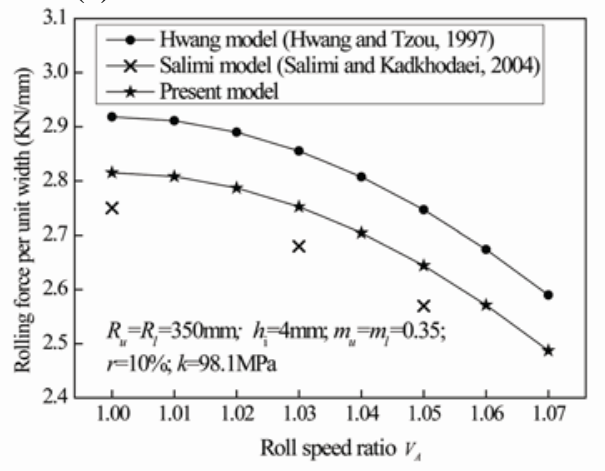

(b)

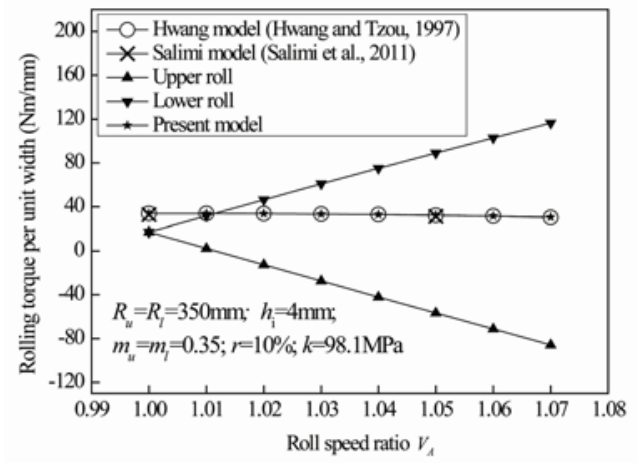

Fig.5 Effect of roll speed ratio on (a) specific rolling force and (b) specific rolling torque

Fig. 5(b) shows the effect of speed ratio on the specific rolling torque. The results of the rolling torque obtained by Hwang and Tzou [3] and Salimi et al. [8] models are also shown in this figure. As the speed ratio increases during asymmetrical rolling process, the location of neutral point of the upper roll increases whereas that of the lower roll decreases. As a result, the rolling torque of the 
lower roll increases while that of the upper roll decreases. Even putridly, the slower roll will be driven by the faster roll and the rolling torque of the slower roll will be negative. Anyway, the total torque calculated by the present model coincides with those of Hwang and Salimi models. They all decrease as the roll speed ratio increases.

Fig. 6(a) illustrates the specific rolling pressure along the contact length with various friction factors $m$. As the $m$ increases, the rolling pressure distribution increases and the cross shear region becomes narrow slightly. Besides, as the $m$ increases both the neutral points $x_{n u}$ and $x_{n l}$ move toward the entrance. Furthermore, sudden changes in the gradient at the location of the two neutral points are due to sudden change in the direction of surface shear stresses at each neutral point.

(a)

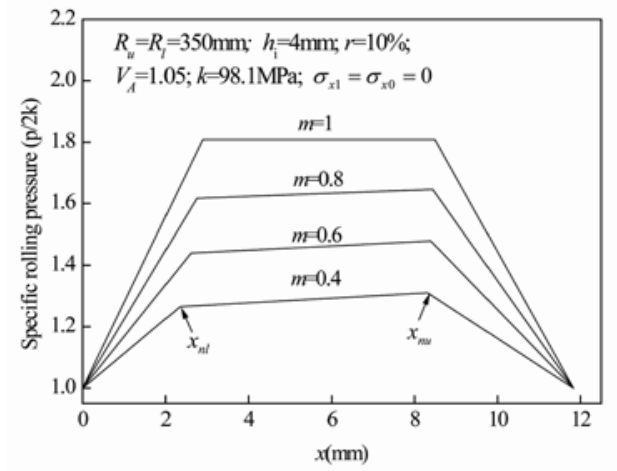

(b)

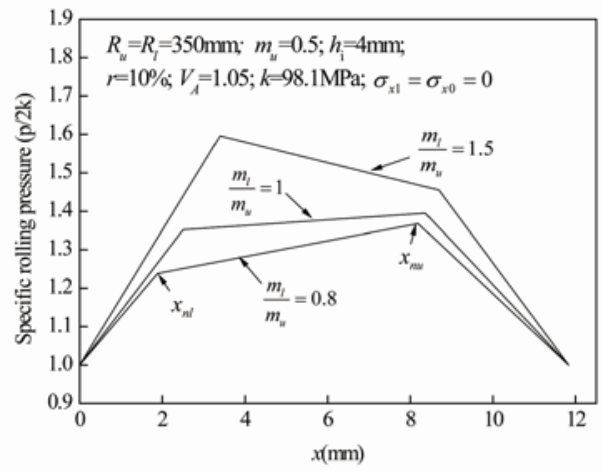

Fig. 6 Variation of specific rolling pressure with (a) various friction factors and (b) various friction factor ratios

Fig. 6(b) shows the variation of rolling pressure with various friction factor ratios $m_{l} / m_{u}\left(m_{u}\right.$ is fixed). Obviously, the whole rolling pressure increases with the increase of $m_{l} / m_{u}$, and the position of neutral points moves toward the entrance of the roll gap as $m_{l} / m_{u}$ increases. The rolling pressure at the neutral point of the upper roll $\left(x_{n u}\right)$ is smaller than at the neutral point of the lower roll $\left(x_{n l}\right)$ in the case of $m_{l} / m_{u}=1.5$, whereas the rolling pressures at $x_{n u}$ are bigger when $m_{l} / m_{u}=1$ and 0.8 .

Fig. 7 shows the effect of input thickness on the rolling force and torque for various speed ratios. It can be seen that, thicker sheets need more rolling force and torque.

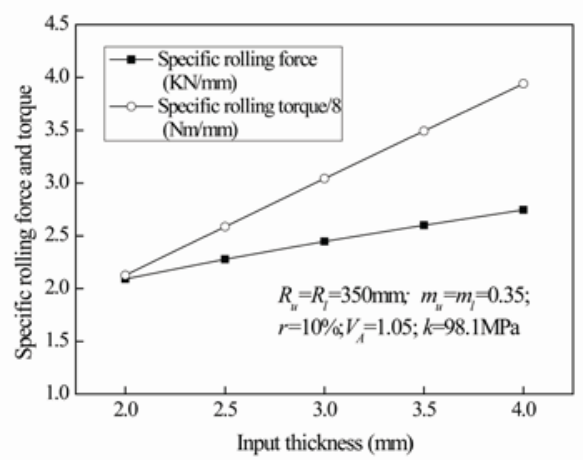

Fig.7 Effect of inlet thickness upon specific rolling force and torque in asymmetrical rolling

Fig. 8 shows the effect of the ratio of work roll radii upon rolling force and torque where the work roll angular speeds are equal. It is seen that by increasing the ratio of work roll radii, the specific rolling force decreases while the specific rolling torque increases. 


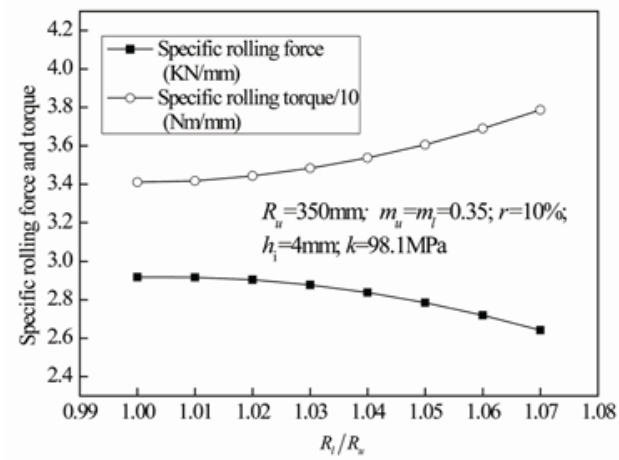

Fig.8 Variations of specific rolling force and torque for equal work roll angular velocities

Fig. 9 shows the specific rolling force and torque with respect to the friction factor ratio. The upper friction factor is assumed to be 0.5 , and the lower one is varied from 0.5 to 1 . Therefore, the asymmetry arises from both speed mismatch and different frictional factors. It is seen that both the specific rolling force and rolling torque increases as the friction factor ratio increases.

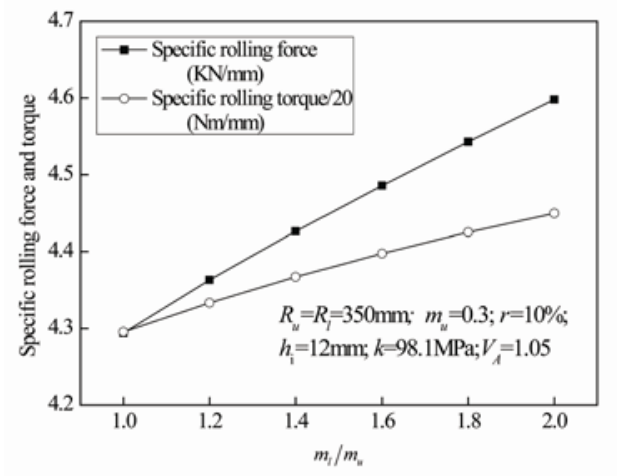

Fig.9 Effect of friction factor ratio on the specific rolling force and torque

\section{Conclusions}

(1) Based on the slab method of analysis, an analytical solution of rolling force and rolling torque considering the shear stresses on the vertical side is first proposed. Comparison of the rolling force and rolling torque obtained from the present model with available data shows a good agreement, and both the specific rolling force and torque decrease as the roll speed ratio increases.

(2) As the $m$ increases, the rolling pressure increases. The position of neutral points moves toward entrance of the roll gap as the $m_{l} / m_{u}$ increases.

(3)Both the rolling force and torque increase with the increase of the plate thickness and the friction factor ratio increases.

(4) The specific rolling force decreases as the ratio of work roll radii increases whereas the trend of the specific rolling torque are reverse.

(5) The present analytical model can easily and quickly predict the characteristics in asymmetrical rolling and is suitable for online control applications.

\section{Acknowledgements}

The authors wish to acknowledge the Basic Research Program of Jiangsu Province (Grant No. BK20140334), the Natural Science Foundation of the Jiangsu Higher Education Institutions of China (Grant No. 14KJB460024) and the Project Funded by China Postdoctoral Science Foundation (Grant No. 2014M561707) . 


\section{References}

[1] G. Sachs, L.J. Klinger: ASME J. Appl. Mech. Vol. 69(1947), p. 88.

[2] D. Pan, D. H. Sansome: Journal of Mechanical Working Technology Vol. 6(1982), p361.

[3] Y. M. Hwang, G. Y. Tzou: Int. J. Mech. Sci. Vol. 39(1997), p289.

[4] M. Salimi, M. Kadkhodaei: J. Mater. Process Technol. 150(2004), p. 215.

[5] M. Kadkhodaei, M. Salimi, M. Poursina: Int. J. Mech. Sci. Vol. 4(2007), p. 622.

[6] P. P. Gudur, M. A. Salunkhe, U. S. Dixit: Int. J. Mech. Sci. Vol. 50(2008), p. 315.

[7] M. Salimi, M. Kadkhodaei: Steel Grips Vol. 150(2004), p. 75.

[8] M. Qwanmizadeh, M. Kadkhodaei, M. Salimi: Int. J. Adv. Manuf. Technol. Vol. 57(2011), p. 5. 